\title{
LESÕES PRECURSORAS E MALIGNAS DE COLO UTERINO - INCIDÊNCIA CONFORME A IDADE
}

\author{
PRE-MALIGNANT AND MALIGNANT CERVICAL LESIONS - \\ INCIDENCE ACCORDING TO AGE
}

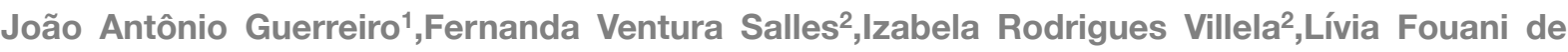
Miranda $^{2}$,Solena Ziemer Kusma ${ }^{3}$

\section{RESUMO}

Objetivo: estabelecer a faixa etária média de diagnóstico de lesões malignas ou pré-malignas de colo de útero, em pacientes atendidas no Hospital Erasto Gaertner, em Curitiba/PR, entre os anos de 2009 e 2011, a fim de comparar estes dados com as diretrizes do Ministério da Saúde. Métodos: estudo retrospectivo de análise de 3136 prontuários de pacientes do Hospital Erasto Gaertner. Foram consultadas outras bibliografias para complementação do estudo. Resultados: a menor média de idade encontrada foi 29,24 anos, sendo 49,25 para câncer invasivo e 34,47 para neoplasia intraepitelial cervical (NIC). O diagnóstico mais encontrado entre as pacientes com menos de 25 anos foi NIC II. Observou-se que a menor média das idades ocorreu em pacientes com NIC I, estando acima de 25 anos e concordando com a literatura de referência. Entretanto, discordando desta, o diagnóstico mais presente em pacientes com menos de 25 anos foi NIC II. Conclusão: ao iniciar o rastreamento aos 25 anos de idade, o diagnóstico da maioria das lesões invasivas e pré-cancerosas seria realizado. Entretanto, existe a possibilidade de subdiagnóstico de NIC II, concordando parcialmente com as recomendações do Ministério da Saúde.

Descritores: Neoplasias de colo de útero. Prevenção de câncer de colo uterino. Teste de Papanicolaou. Esfregaço vaginal. Neoplasia intraepitelial cervical.

\section{ABSTRACT}

Objective: to establish the mean age at diagnosis of malignant or pre-malignant cervical lesions in patients treated at Erasto Gaertner Hospital, in Curitiba/PR, between the years of 2009 and 2011, in order to compare these data with the guidelines of the Brazilian Ministry of Health. Methods: retrospective analysis of 3136 medical records of patients from Erasto Gaertner Hospital. Alternative sources were consulted for the complementation of the study. Results: The lowest average age was 29,24 years, being 49,25 for invasive cancer and 34,47 for cervical intraepithelial neoplasia (CIN). Only $0.4 \%$ of patients with invasive cancer were younger than 25 years. The most common diagnosis among patients younger than 25 years was CIN II. It was observed that the lower average age occurred in patients with CIN I, and it was over 25 years, therefore agreeing with literature. However, disagreeing with this, the most common diagnosis in patients under 25 years was CIN II. Conclusion: if the screening is initiated at 25 years old, the majority cases of malignant and premalignant cervical lesions would have been diagnosed. However, there is a possibility of under-diagnosis of CIN II, partially agreeing with the recommendations of the Ministry of Health.

Keywords: Uterin cervical neoplasms. Cervix neoplasm prevention. Papanicolaou test. Vaginal smears. Cervical intraepithelial neoplasia.

1 - Médico Cirurgião Oncológico do Hospital Erasto Gaertner e professor da disciplina de Oncologia da Pontifícia Universidade Católica do Paraná

2 - Graduandas do curso de Medicina da Pontifícia Universidade Católica do Paraná

3 - Doutora em Saúde Pública pela Pontifícia Universidade Católica do Paraná

Contato do Autor / Mail to:

Izabela Rodrigues Villela - iza_villela@hotmail.com

Rua Doutor Ovande do Amaral, 201 - Jardim das Américas, Curitiba, Paraná, Brasil - CEP 81.520-060 


\section{INTRODUÇÃO}

O câncer cervicouterino é o quarto tipo de neoplasia mais comum entre as mulheres no mundo, sendo o terceiro mais comum entre as brasileiras. Anualmente, esta doença corresponde a 530 mil novos casos e a 265 mil óbitos no mundo. A sua incidência aumenta gradativamente, com maior taxa entre 45 e 50 anos, havendo maior mortalidade a partir da quarta década de vida ${ }^{1,2}$.

Esta neoplasia possui evolução lenta e progressiva, iniciando com fases pré-invasivas e benignas, as Neoplasias Intraepiteliais Cervicais (NIC). O exame citopatológico (colpocitologia oncótica ou Papanicolaou) possibilita o rastreamento e o tratamento precoces das lesões ${ }^{1}$.

Entre os fatores de risco da doença estão: idade, raça negra, tabagismo, não realização do exame Papanicolaou, início precoce da atividade sexual, múltiplos parceiros, imunossupressão, baixa condição socioeconômica e, principalmente, infecção prévia pelo Papilomavírus humano (HPV), sendo os mais oncogênicos os tipos 16 e 18 ${ }^{1,3-5}$.

O câncer de colo uterino possui início assintomático porém, com o seu desenvolvimento, pode haver queixas de dispareunia, leucorreia, sinusiorragia e sangramento vaginal. Em estágios mais avançados, podem surgir sintomas consumptivos e manifestações causadas pela invasão e obstrução de estruturas vizinhas, como dor pélvica ou lombar e perda de urina ou fezes pela vagina ${ }^{3,5}$.

Ações preventivas possibilitam índice de cura próximo a $100 \%$ quando há o diagnóstico precoce. Dentre elas, incluem-se: uso de preservativos, rastreamento com exame Papanicolaou e tratamento de lesões em estágios iniciais ${ }^{1}$.

A introdução da citologia do colo uterino no Brasil ocorreu em 1942. e consiste na coleta e análise de células da ectocérvice e da endocérvice, através da raspagem do colo uterino. É considerada eficaz, com sensibilidade de $70 \%$ e especificidade de 90 a $99 \%$. Em 1996, o Ministério da Saúde inseriu o Programa Viva Mulher, visando o desenvolvimento de protocolos para a coleta e análise do exame citopatológico. Em 1998, instituiu-se o Programa Nacional de Combate ao Câncer de Colo de Útero ${ }^{6-8}$.

Em 2011, o Ministério da Saúde introduziu as Diretrizes Brasileiras para o Rastreamento do Câncer de Colo de Útero, visando padronizar as recomendações para investigação do câncer cervicouterino, sendo estas:

A. O exame Papanicolaou seja realizado trienalmente, após dois exames anuais negativos;
B. A coleta seja iniciada aos 25 anos em mulheres que já iniciaram a vida sexual;

C. Os exames sejam continuados até 64 anos de idade e devem ser interrompidos após esta idade quando dois exames consecutivos forem negativos nos últimos cinco anos;

D. Em mulheres com mais de 64 anos que nunca realizaram o exame citopatológico, deva ser realizados dois exames com intervalo de um a três anos entre eles. Se forem negativos, não serão necessários exames posteriores ${ }^{6-8}$.

Assim, o presente estudo tem como objetivo estabelecer a faixa etária média de diagnóstico de lesões malignas ou pré-malignas de colo uterino em pacientes atendidas no Hospital Erasto Gaertner entre os anos de 2009 e 2011, a fim de comparar estes dados com as recomendações a respeito da idade de início do rastreamento das Diretrizes do Ministério da Saúde.

\section{MÉTODOS}

Trata-se de um estudo retrospectivo realizado entre novembro de 2013 e novembro de 2014. Foram analisados 3136 prontuários eletrônicos do Hospital Erasto Gaertner de pacientes que tinham diagnóstico de câncer cervicouterino ou de lesões precursoras. Foram coletados os dados: diagnóstico, idade no momento do diagnóstico e ano em que ele foi feito.

Os critérios de inclusão foram: pacientes do sexo feminino diagnosticadas com carcinoma escamocelular (CEC) de colo uterino, adenocarcinoma, NIC I, II ou III no Hospital Erasto Gaertner, entre 01 de janeiro de 2009 e 31 de dezembro de 2011.

Os critérios de exclusão foram: pacientes que não apresentavam lesão maligna ou precursora de câncer cervicouterino, pacientes que não foram diagnosticadas nos anos de 2009, 2010 e 2011 ou com outro tipo de câncer, e mulheres que tiveram o diagnóstico em outra instituição e não realizaram a citologia oncótica no Hospital Erasto Gaertner para confirmação. Assim, foram excluídos 636 e utilizados 2497 prontuários.

Os dados foram armazenados em planilhas do Microsoft Excel $^{\circledR}$ e analisados estatisticamente pelo programa computacional SPSS versão 20.0.

Para revisão da literatura e discussão, foram analisadas publicações indexadas nas bases Medical Literature Analysis and Retrieval System Online (MEDLINE), por meio do PubMed, Literatura Latinoamericana e do Caribe em Ciências da Saúde (Lilacs), 
Cochrane e Scientific Eletronic Library Online (SciELO), a partir de 2008 .

Foram selecionadas 17 referências bibliográficas, entre elas, nove artigos, três livros da biblioteca do Hospital Erasto Gaertner e duas diretrizes, uma do Ministério da Saúde e outra do Instituto Nacional de Câncer (INCA). Também foram consultadas duas Cartilhas, uma do Programa Viva Mulher e outra do Ministério da Saúde, além de dados do site do INCA.

As pacientes não foram abordadas para a confecção desse trabalho, sendo este exclusivamente uma análise de prontuário médico, prezando o anonimato e respeitando o termo de confidencialidade assinado pelos autores.

Este estudo foi conduzido conforme os preceitos éticos determinados pelo Conselho Nacional de Saúde do Ministério da Saúde. O projeto de pesquisa foi submetido ao Comitê de Ética em Pesquisa do Hospital Erasto Gaertner, sendo aprovado pelo CAAE 32327014.3.0000.0098, com parecer $n^{\circ}$ 2332. Não houve conflito de interesses.

\section{RESULTADOS}

Dos 2497 prontuários analisados, 629 eram do ano de 2009, 1010 de 2010 e 858 de 2011, com idade das pacientes entre 13 e 101 anos, fornecendo média de idade de 37,97 anos e mediana de 35,00 anos, como mostra a tabela 1.

Tabela 01 - Média, desvio padrão, mínima e máxima das idades das pacientes

\begin{tabular}{|c|c|c|c|c|c|}
\hline $\begin{array}{c}\text { Diagnós } \\
\text { tico }\end{array}$ & $\begin{array}{l}\text { Frequ } \\
\text { ência }\end{array}$ & $\begin{array}{c}\text { Média } \\
\text { de } \\
\text { Idade } \\
\text { (anos) }\end{array}$ & $\begin{array}{c}\text { Desvio } \\
\text { Padrã } \\
\text { o } \\
\text { (anos) }\end{array}$ & $\begin{array}{c}\text { Idade } \\
\text { Míni } \\
\text { ma } \\
\text { (anos } \\
\text { ) }\end{array}$ & $\begin{array}{c}\text { Idade } \\
\text { Máxi } \\
\text { ma } \\
\text { (anos) }\end{array}$ \\
\hline $\begin{array}{l}\text { Adenoca } \\
\text { rcinoma }\end{array}$ & 84 & 47,44 & 13,662 & 27 & 84 \\
\hline$C E C$ & 506 & 49,55 & 14,869 & 19 & 101 \\
\hline NIC I & 362 & 29,24 & 10,663 & 14 & 78 \\
\hline NIC II & 967 & 34,73 & 11,811 & 13 & 83 \\
\hline NIC III & 578 & 37,32 & 12,149 & 17 & 86 \\
\hline Total & 2497 & 37,97 & 14,175 & 13 & 101 \\
\hline
\end{tabular}

Fonte: os autores, 2014.
Os dados relacionados ao aspecto socioeconômico das famílias também foram abordados por meio dos questionários. Como resultado, 10\% das mães tinham menos de 20 anos de idade e 7,7\% delas haviam estudado por menos de 5 anos. A renda familiar de $79,2 \%$ das participantes era inferior a 3 salários mínimos (Tabela 1).

Nas pacientes diagnosticadas com NIC I, a média de idade foi de 29,24 anos, com NIC II foi de 34,73 anos, com NIC III foi de 37,32 anos, com adenocarcinoma foi de 47,44 anos e com CEC foi de 49,55 anos.

Dentre as pacientes com NIC I, a idade mínima encontrada foi de 14 anos e a máxima de 78 anos; com NIC II, a mínima foi de 13 e a máxima de 83 anos; com NIC III, a mínima foi de 17 e a máxima de 86 anos. Já entre as pacientes com adenocarcinoma, a menor idade foi de 27 anos e a maior de 84 anos e com CEC, a mínima foi de 19 e a máxima de 101 anos.

Existiam 590 pacientes com o diagnóstico de câncer invasivo de colo uterino, com média de idade de 49,25 anos e 1907 com diagnóstico de NIC, com média de 34,47 anos. O desvio padrão da média das idades dos casos invasivos foi de 14,71 anos e dos casos de NIC foi de 12,02 anos. Na análise do t-test, observouse significância entre as médias das idades entre os casos malignos e de NIC $(p<0,01)$, conforme evidencia a tabela 2 .

Tabela 02 - Comparação entre as médias das idades de acordo com o diagnóstico

\begin{tabular}{lccc}
\hline Diagnóstico & $\begin{array}{c}\text { Frequên } \\
\text { cia (n) }\end{array}$ & $\begin{array}{c}\text { Média de } \\
\text { idade } \\
\text { (anos) }\end{array}$ & $\begin{array}{c}\text { Desvio } \\
\text { Padrão }\end{array}$ \\
\hline $\begin{array}{l}\text { Câncer } \\
\text { invasivo }\end{array}$ & 590 & 49,25 & 14,711 \\
$\begin{array}{l}\text { Neoplasia } \\
\text { intraepitelial }\end{array}$ & 1907 & 34,47 & 12,026 \\
\hline$p<0,01$ & & &
\end{tabular}

Fonte: os autores, 2014. 
Do total dos pacientes, $14,5 \%$ possuem NIC I, sendo 5,8\% com menos de 25 anos e $8,7 \%$ com idade igual ou superior a 25 anos; 38,7\% apresentam NIC II, sendo $6,5 \%$ com menos de 25 anos e $32,2 \%$ com 25 anos ou mais; e $23,1 \%$ foram diagnosticados com NIC III, sendo $2,1 \%$ com menos de 25 anos e $21,1 \%$ com 25 anos ou mais, como mostram as tabelas 3 e 4 .

Tabela 03 - Frequência dos diagnósticos

\begin{tabular}{lcc}
\hline \multicolumn{1}{c}{ Diagnóstico } & Frequência & $\begin{array}{c}\text { Percentual } \\
\mathbf{( \% )}\end{array}$ \\
\hline Adenocarcinoma & 84 & 3,4 \\
CEC & 506 & 20,3 \\
NIC I & 362 & 14,5 \\
NIC II & 967 & 38,7 \\
NIC III & 578 & 23,1 \\
Total & 2497 & 100,0 \\
\hline
\end{tabular}

Fonte: os autores, 2014.

Tabela 04 - Comparação dos diagnósticos de acordo com a idade ( 25 anos)

\begin{tabular}{|c|c|c|c|}
\hline \multirow[b]{2}{*}{$\begin{array}{c}\text { Diagnóstico } \\
\text { Final }\end{array}$} & \multicolumn{2}{|c|}{ Idade } & \multirow[b]{2}{*}{$\begin{array}{l}\text { Total } \\
\text { n (\%) }\end{array}$} \\
\hline & $\begin{array}{c}\text { Inferior a } \\
25 \text { anos } \\
\text { n (\%) }\end{array}$ & $\begin{array}{c}25 \text { anos } \\
\text { ou mais } \\
\text { n (\%) }\end{array}$ & \\
\hline $\begin{array}{l}\text { Adenocarcinom } \\
a\end{array}$ & $0(0,0 \%)$ & $84(3,9 \%)$ & $\begin{array}{c}84 \\
(3,9 \%)\end{array}$ \\
\hline$C E C$ & $9(2,5 \%)$ & $\begin{array}{c}497 \\
(23,3 \%)\end{array}$ & $\begin{array}{c}506 \\
(20,3 \%)\end{array}$ \\
\hline$N I C I$ & $\begin{array}{c}144 \\
(39,2 \%)\end{array}$ & $\begin{array}{c}218 \\
(10,2 \%)\end{array}$ & $\begin{array}{c}362 \\
(14,5 \%)\end{array}$ \\
\hline NIC II & $\begin{array}{c}162 \\
(44,1 \%)\end{array}$ & $\begin{array}{c}805 \\
(37,8 \%)\end{array}$ & $\begin{array}{c}967 \\
(38,7 \%)\end{array}$ \\
\hline NIC III & $52(14,2 \%)$ & $\begin{array}{c}526 \\
(24,7 \%)\end{array}$ & $\begin{array}{c}578 \\
(23,1 \%)\end{array}$ \\
\hline Total & $\begin{array}{c}367 \\
(100,0 \%)\end{array}$ & $\begin{array}{c}2130 \\
(100,0 \%)\end{array}$ & $\begin{array}{c}2497 \\
(100,0 \% \\
)\end{array}$ \\
\hline
\end{tabular}

$p<0,01$

Fonte: os

autores, 2014.
Das 2497 pacientes, 3,4\% apresentavam adenocarcinoma, sendo que nenhuma possui idade inferior a 25 anos, e 20,3\% têm CEC, sendo 0,4\% com menos de 25 anos e 19,9\% com 25 anos ou mais.

Foram diagnosticadas 367 pacientes com menos de 25 anos (14,7\% do total), dentre elas: $39,2 \%$ com NIC I, 44,1\% com NIC II, 14,2\% com NIC III, 2,5\% com CEC e nenhuma com adenocarcinoma.

Do total de pacientes, 2130 possuíam 25 anos ou mais, sendo $10,2 \%$ com NIC I, 37,8\% com NIC II, $24,7 \%$ com NIC III, 23,3\% com CEC e 3,9\% com adenocarcinoma.

$\mathrm{Na}$ comparação entre os diagnósticos conforme a faixa etária, foi encontrada diferença significante entre a incidência das patologias segundo a idade das pacientes $(p<0,01)$.

Dentre todas as pacientes, apenas 152 possuíam 65 anos ou mais, representando $6,1 \%$. Destas, $2 \%$ possuíam NIC I, 16,4\% NIC II, 17,8\% NIC III, 8,6\% adenocarcinoma e 55,3\% CEC. De acordo com a análise realizada, foi constatada uma diferença significativa entre as idades conforme os diagnósticos $(p<0,01)$, como mostra a tabela 5 .

Tabela 05 - Comparação dos diagnósticos de acordo com a idade (65 anos)

\begin{tabular}{lccc}
\cline { 1 - 2 } Diagnóstic & \multicolumn{2}{c}{ Idade } & \\
\cline { 2 - 3 } o Final & Idade $<\mathbf{6 5}$ & Idade $\geq \mathbf{6 5}$ & Total \\
& $\mathbf{n}(\%)$ & $\mathbf{n}(\%)$ & $\mathbf{n}(\%)$ \\
\hline Adenocarci & $71(3.0 \%)$ & $13(8,6 \%)$ & $84(3,4 \%)$ \\
noma & & & \\
CEC & $422(18,0 \%)$ & $84(55,3 \%)$ & $506(20,3 \%)$ \\
NIC I & $359(15,3 \%)$ & $3(2,0 \%)$ & $362(14,5 \%)$ \\
NIC II & $942(40,2 \%)$ & $25(16,4 \%)$ & $967(38,7 \%)$ \\
NIC III & $551(23,5 \%)$ & $27(17,8 \%)$ & $578(23,1 \%)$ \\
Total & 2345 & 152 & 2497 \\
\hline$p<0,01$ & $(100,0 \%)$ & $(100,0 \%)$ & $(100,0 \%)$ \\
\hline
\end{tabular}

Fonte: os autores, 2014. 


\section{DISCUSSÃO}

Diversos métodos estão disponíveis para a detecção precoce do câncer de colo uterino. A realização periódica do exame citopatológico com técnica de Papanicolaou é considerada a melhor forma de rastreamento ${ }^{6-10}$.

Ainda hoje, o Brasil apresenta uma taxa de cobertura inferior à recomendada pela Organização Mundial de Saúde (OMS), que é de $80 \%$ da população feminina entre 35 e 59 anos. Este dado justifica a maior incidência de doença invasora em regiões menos desenvolvidas e com acesso precário à rede de saúde, representando uma importante causa de óbito entre as mulheres nestes locais $4,8,9,11,12,13$.

Diversos estudos identificaram que a incidência e a mortalidade do câncer cervicouterino não se alteram significativamente ao iniciar o rastreamento da doença em mulheres com menos de 25 anos. Um artigo publicado pela International Agency for Research on Cancer (IARC) em 1986 observou que a incidência cumulativa da neoplasia de câncer cervicouterino aos 25 anos, ao invés de aos 20 anos, diminui apenas em $1 \%$.

A análise mostrou que a menor média das idades ocorreu em pacientes com NIC I (29,24 anos), sendo que este valor encontra-se acima de 25 anos, idade indicada para início do rastreamento pelas Diretrizes do Ministério da Saúde ${ }^{6}$.

No presente artigo, foi encontrada nas pacientes com neoplasias invasoras uma média de idade de 49,25 anos, com desvio padrão de 14,71 anos. No estudo de Coelho e Costa, realizado no Hospital A. C. Camargo em 2000, foram encontrados valores semelhantes, com média de idade de 48,5 anos e desvio padrão de 14,3 anos $^{14}$.

Das 2497 pacientes analisadas neste estudo, 367 apresentavam menos de 25 anos. Destas, apenas 0,4\% possuíam câncer invasivo. Um estudo com base no Registro Hospitalar de Câncer da Fundação Oncocentro de São Paulo (FOSP), apenas 1,03\% dos casos de câncer invasor foi encontrado em mulheres com menos de 25 anos. Concordando com este dado, em uma análise realizada pela Unicamp somente $1,12 \%$ dos pacientes com câncer invasor tinham até 24 anos $^{6}$.

Também segundo as Diretrizes, grande parte dos diagnósticos em mulheres abaixo dos 25 anos é de NIC I. Entretanto, no presente estudo, viu-se que a maioria das pacientes abaixo de 25 anos tiveram diagnóstico de NIC $\|^{4,6}$.

Conforme as Diretrizes Brasileiras para o Rastreamento do Câncer do Colo do Útero, o rastreamento em mulheres com menos de 25 anos faz com que sejam diagnosticadas mais lesões de baixo grau, que não são consideradas precursoras. Com isso, mais procedimentos e tratamentos desnecessários são instituídos. A mesma ressalta que o tratamento de lesões em mulheres jovens está relacionado à maior morbidade obstétrica e a maiores taxas de partos prematuros, sendo justificável a diminuição de intervenções nestes casos ${ }^{6}$.

Outra razão que justifica a não realização do exame citopatológico em mulheres com menos de 25 anos é a evidência de que este é menos eficaz na detecção de lesões nesta faixa etária. O impacto psíquico ao diagnosticar-se uma lesão precursora de câncer também foi levado em consideração pelas Diretrizes ao não indicar o Papanicolaou antes dos 25 anos $^{6}$.

Existem menos evidências sobre qual a idade ideal para o término do rastreamento. Há uma propensão em aumentar o intervalo entre os exames em mulheres de maior idade, dado que não existem informações objetivas sobre a utilidade de tais procedimentos em pacientes com 65 anos ou mais. Neste artigo apenas 152 das 2497 pacientes possuíam idade igual ou superior a 65 anos, sendo que, destas, $55,3 \%$ tinham CEC 6 .

\section{CONCLUSÃO}

Observou-se que os dados obtidos neste estudo concordam em parte com os argumentos presentes nas Diretrizes. De fato, foi encontrada uma taxa pequena de casos invasivos em mulheres com menos de 25 anos e, também, a menor média de idade de diagnóstico encontrada foi de 29,24 anos.

Entretanto, nas pacientes com menos de 25 anos, a maioria dos casos era de NIC II. Deve-se levar em conta que a NIC II é uma lesão precursora de câncer cervicouterino, devendo ser tratada e acompanhada, não podendo deixar de ser realizado o seu diagnóstico precoce.

Conclui-se que ao iniciar o rastreamento aos 25 anos de idade, o diagnóstico da maioria das lesões invasivas e pré-cancerosas seria realizado. Mas, existe a possibilidade de subdiagnóstico de lesões NIC II.

Para melhores análises, sugerem-se futuros estudos focando na incidência das lesões de alto grau em pacientes com menos de 25 anos, uma vez que o presente trabalho apontou que esta taxa não é insignificante. 


\section{REVISTA MÉDICA DA UFPR}

\section{REFERÊNCIAS}

1. Silva MB da, Nascimento MC do, Ribeiro DA de A, Matias IM de S, Gradim CVC. Rastreamento do câncer de colo de útero em uma Unidade Básica de Saúde do Estado de Minas Gerais. Cad. Saúde Coletiva 2012; 20(3):265-270.

2. Instituto Nacional de Câncer. Controle do Câncer do Colo do Útero. 2014. Available at: http:// www2.inca.gov.br/wps/wcm/connect/ acoes_programas/site/home/nobrasil/ programa_nacional_controle_cancer_colo_utero/ conceito_magnitude. Acessado em julho de 2014.

3. Diz MDPE, Medeiros RB de. Câncer de colo uterino - fatores de risco, prevenção, diagnóstico e tratamento. Rev Med 2009;88(1):7-15.

4. Araujo SR. Citologia Cérvico-Vaginal Passo a Passo. 22a Edição. Curitiba, Paraná; 2010.

5. Guimarães JRQ. Manual de Oncologia. 3a Edição. (Editora B, ed.). São Paulo, SP; 2008.

6. Ministério da Saúde, Instituto Nacional de Câncer. Diretrizes Brasileiras para o Rastreamento do Câncer do Colo do Útero. 2011.

7. Ministério da Saúde. Falando Sobre Câncer do Colo do Útero. 2002:1-50.

8. Corrêa DAD. Perfil das usuárias do Sistema Único de Saúde que realizam o Papanicolaou em Manaus, Amazonas. 2009.

9. Caetano R, Vianna CM de M, Thuler LCS, Girianelli VR. Custo-efetividade no diagnóstico precoce do câncer de colo uterino no Brasil. Rev Saude Coletiva 2006;16(1):99-118.

10. Pinho A de A, França-Junior I. Prevenção do câncer de colo do útero: um modelo teórico para analisar o acesso e a utilização do teste de Papanicolaou. Rev. Bras. Saúde Matern. Infant. 2003;3(1):95-112.

11. Secretaria Municipal de Saúde de Curitiba. Programa Viva Mulher Em Curitiba: Controle Do Câncer de Mama E Colo de Útero. Curitiba; 2002.

12. Zeferino LC. O desafio de reduzir a mortalidade por câncer do colo do útero. Rev. Bras. Ginecol. e Obstet. 2008;30(5):213-215.

13. Thuler LCS. Mortalidade por câncer do colo do útero no Brasil. Rev. Bras. Ginecol. e Obstet. 2008;30(5):216-218.
14. Coelho FRG, Costa RLR. Padronização Em Ginecologia Oncológica. São Paulo, SP; 2005. 